\title{
Blistering and Hydraulic Removal of Scale Films of \\ Rimmed Steel at High Temperature*
}

\author{
By Fumio MATSUNO**
}

\begin{abstract}
Synopsis
Under the protection from the oxidation, rimmed steel plates were heated to temperatures in the range $800^{\circ} \sim 1250^{\circ} \mathrm{C}$. Then they were oxidized in air for very short periods. Blistering and hydraulic removal of scale films of them were studied.

Blisters, which were caused by the compressive stresses such as the oxidation stress and the thermal stress, were small round spots at first, and they grew rapidly. The periods from the start of oxidation to the appearance of blisters were measured at various conditions.

The removability of scale films was examined by the water jets with pressures of 1,4 and $15 \mathrm{~kg} / \mathrm{cm}^{2}$. Scale films with some blisters were removed readily by the water jets.

Based on the above results the situation of the stress in the scale film and its influences on the hydraulic removal were discussed.
\end{abstract}

\section{Introduction}

During the continuous rolling of steel strip, the formation of surface oxides can lead to defects in the finished strip. To prevent these defects, it is essential that the oxides are efficiently removed. For this purpose, a typical rolling schedule for steel strip includes mechanical and hydraulic descaling before the first rougher descaling sprays in the roughing train and a high-pressure spray bank before the finishing train.

Although there is a comprehensive literature ${ }^{1,2)}$ on the mechanism of oxidation and subsequent structure of scale, there are few reported works on scale removal at high temperatures by hydraulic descaling which is a common process in the steel industry. According to Sheppard and Steen, ${ }^{3)}$ the total stress produced in the scale by descaling is the product of the thermal stress produced by the cooling action of water jets, the stress due to the impact force of the jet, and the depositional compression stress (the oxidation stress). Any one of these or any combination of stresses could be process controlling. The present work was intended to investigate qualitatively the relation between the degree of the oxidation stress and the hydraulic removal of scale films formed during the hot rolling at various oxidation temperatures and periods in air.

There have been reported lots of works on the stress generation during scale formation. ${ }^{4-21)}$ Pilling and Bedworth ${ }^{4}$ were among the first investigators to recognize the importance of the oxidation stress in the scaling process. They postulated that the stress arose as a result of volume dilatation upon the conversion of metal to its oxide. The ratio of volume of oxide formed to the corresponding volume of metal consumed has become known as the Pilling-Bedworth Ratio (PBR). If the PBR $>1$, the oxide would be put into compression and the metal in tension, and vice versa for $\mathrm{PBR}<1$. If the $\mathrm{PBR}$ is unity, the system is free from stress. In practice, it has been found that the PBR gives an indication of the sign but not the magnitude of stress system.

The formation of oxide scales of various metals and alloys is generally believed to proceed according to the Wagner mechanism ${ }^{22}$ ) wherein material is transported by diffusion across layers of reaction products. It can easily be seen that, if vacancy diffusion and condensation is insufficiently rapid to provide all the space for the growing oxide, then stresses must be generated during anion diffusion-controlled oxidation. During cation diffusion-controlled oxidation, however, there must always be an ample space available for the new oxide at the oxide-atmosphere interface. Nevertheless, stress is generated during the oxidation of metals which are known to be oxidized predominantly by cation diffusion. In order to explain this, Jaenicke et al. ${ }^{13)}$ have considered that the stress arises from the growth of new oxide in cracks or open pores in the scale film. Rhines and Wolf ${ }^{12)}$ have proposed that some anion diffusion occurs down the oxide grain boundary, and a chemical reaction takes place with those cations which happen to intersect these boundaries during their outward journey through the oxide lattice. Thus, it was argued, new oxide forms within the scale along the boundary giving rise to compressive stresses. This theory has received wide spread support and it has been applied, for example, for the explanation of the stability of scales of heat resisting steels at elevated temperatures. ${ }^{23)}$ Harris and Speight, ${ }^{20,21)}$ however, have the following opinions. The location for the formation of new oxide depends on the relative rates of diffusion of anions and cations, not on the absolute rate of diffusion of either species. Implicit in the Rhines and Wolf model is the assumption that the rate of anion diffusion down the grain boundary exceeds (or is approximately equal to) the rate of cation diffusion up the boundary. This would contrast with the relative rates within the oxide grain boundaries where cation diffusion is faster (otherwise the oxidation process would not have been termed "cation controlled "). It must be considered highly unlikely that the relative rates should be reversed upon moving from intragranular diffusion to intergranular diffusion. On the basis of the above discussion, Harris and Speight ${ }^{20,21)}$ have considered that the mechanism proposed by Jaenicke et al. ${ }^{13)}$ is more probable.

* Originally published in Tetsu-to-Hagané, 65 (1979), 599, in Japanese. English version received August 22, 1979.

** Central Research Laboratories, Sumitomo Metal Industries, Ltd., Nishinagasu-hondori, Amagasaki 660. 
Although no single concept of the origin of the oxidation stress has yet attained general acceptance as mentioned above, it is undoubted that the stress generation takes place during the oxidation. Many experimental evidences have been reported. In case of the oxidation of iron, the stress generation has been confirmed by Engell and Wever ${ }^{19)}$ who observed that helical specimens of iron tended to "wind up" during high temperature oxidation. As PBR of iron-FeO system is 1.78 and that of iron- $\mathrm{Fe}_{3} \mathrm{O}_{4}$ system is $2.1,{ }^{24)}$ iron oxide is put into compression and iron in tension.

The oxidation stress generated has been evaluated by several methods using the deformation behaviors of specimens such as creep and bending caused by it. However, these methods are difficult to apply in the present study intending to investigate the scale films formed during hot rolling, because the formation of scales takes place at too high temperatures and for too short time to use the reported methods. By the way, blisters of scale films have often been provided as an evidence that the stress is present in the scale. So, in this study, blistering of scale films at elevated temperatures was chosen as an index for the evaluation of the oxidation stress. However, blisters have also been reported to be due to gas bubbles released from steel or formed by decarburization. ${ }^{25,26)}$ For this reason, a preliminary study was also carried out in order to confirm the motive force causing blistering of the scale film of the rimmed steel examined. The effect of thermal stress on blistering was also studied because hot rolling of steels is usually carried out during the cooling in air.

In addition to the observation of blistering, hydraulic descaling of scale films formed at various conditions was studied by the method analogous to that of Sheppard and Steen. ${ }^{3)}$ And the relation between the degree of the oxidation stress and the removability of scale films by water jets was discussed.

\section{Experiments}

A rimmed steel was mostly used and its chemical compositions are presented in Table 1. In order to study the properties of scales formed during hot rolling, following studies were carried out.

\section{Growth Rate and Microstructure of Scale Film}

Specimens of size $20 \times 10 \times 3 \mathrm{~mm}$ were abraded on successively finer $\mathrm{SiC}$ papers to 600 grade. They were degreased by petroleum benzine prior to oxidation. Preweighed specimens were set on a support made by 18-8 stainless steel and put into a bakeable vacuum apparatus. After evacuation to about $10^{-3}$ Torr specimens were preheated to reaction temperatures in vacuum. Then, air was admitted into the

Table 1. Chemical compositions of the rimmed steel examined. (wt \%)

\begin{tabular}{ccccccc}
\hline C & Si & Mn & P & S & Cu & Cr \\
\hline 0.08 & 0.01 & 0.36 & 0.008 & 0.020 & 0.05 & 0.02 \\
\hline
\end{tabular}

quartz reaction tube. Oxidation was carried out at $950^{\circ}, 1000^{\circ}, 1050^{\circ}, 1100^{\circ}$ and $1150^{\circ} \mathrm{C}$ for 30 , 60, 90, 120 and $150 \mathrm{sec}$, respectively. The runs were terminated by the reevacuation and the rapid cooling of the reaction tube with water spray after taking out it from the furnace. Specimens were weighed and the oxidation weight gains were determined.

The microstructures of not only specimens obtained by the above methods, but those cooled from the reaction temperature in air were examined by the usual microscopic observation and the X-ray diffraction technique.

\section{Observation of Blistering of Scale Film}

\section{Preliminary Study}

Gas bubbles were reported to be due to blisters in addition to the oxidation stress. ${ }^{25,26)}$ Following experiments were carried out to confirm whether gas bubbles are the origin of blisters or not in case of the rimmed steel used in this study.

Pure iron containing less than $0.001 \% \mathrm{C}$ was prepared in addition to the rimmed steel used. Specimens, nominally $50 \times 50 \times 3 \mathrm{~mm}$, were put into a bakeable vacuum apparatus and heated to $900^{\circ} \mathrm{C}$ in vacuum of $10^{-4}$ Torr. After keeping at that temperature in vacuum for $60 \mathrm{~min}$., they were oxidized in air and blistering of scale films of both specimens was observed.

\section{Observation of Blistering of Rimmed Steel at the Iso- thermal Oxidation}

Specimens of size $50 \times 50 \times 3 \mathrm{~mm}$ were prepared from a hot-rolled rimmed steel strip which was pickled by hydrochloric acid. For the purpose of the protection from the oxidation during the preheating, one of surfaces of them was covered with a cold-rolled rimmed steel sheet of $0.6 \mathrm{~mm}$ thick. Specimens were put into a tubular furnace heated at the desired temperatures. Both sides of the alumina furnace tube were closed by caps made by refractory bricks. Ar gas was admitted into the tube during the preheating of specimens. Temperature of specimens was measured by a Pt-Pt 13\% Rh thermo-couple of $0.2 \mathrm{~mm}$ in diameter attached to its surface. After temperatures were reached to the desired ones in the range $800^{\circ} \sim 1250^{\circ} \mathrm{C}$, admittance of Ar gas was terminated and the substitution of furnace atmosphere from Ar to air was carried out sufficiently by opening the both sides of the furnace tube. Then, the tube was reclosed in order to avoid the fluctuation of the temperature during the oxidation. The temperature of the furnace was controlled automatically within $\pm 3^{\circ} \mathrm{C}$. Oxidation was started by the removal of the steel sheet cover by means of pulling a nicrome wire attached to it. Blistering of scales was observed from a hole of about $5 \mathrm{~mm}$ in diameter drilled in one of the caps.

\section{Observation of Blistering during the Cooling in Air}

In order to get various cooling rates during the oxidation specimens of size $100 \times 100 \times 3,100 \times$ $100 \times 10, \quad 100 \times 100 \times 20$ and $100 \times 100 \times 30 \mathrm{~mm}$ were used. Heating of specimens was carried out by the same method mentioned above. After temperatures 
were reached to the desired ones in the range $850^{\circ} \sim$ $1250^{\circ} \mathrm{C}$, specimens were taken out from the furnace and placed on a refractory brick. Oxidation was started by the removal of the steel sheet covered the upper surface of specimen and blistering of scale films was observed. Additionally, thermal shock was given to some of the specimens, which had still no blisters, by a local gas spraying on their surface and the effects of thermally induced stress on blistering were studied.

\section{Descaling by Water Jets}

Specimens of size $100 \times 100 \times 30 \mathrm{~mm}$ were heated by the same method mentioned above. After temperatures were reached to the desired ones in the range $800^{\circ} \sim 1200^{\circ} \mathrm{C}$, they were taken out from the furnace and placed on a brick of the apparatus shown in Fig. 1. Then, oxidation was started by the removal of the steel sheet cover. After the oxidation for various periods from $5 \mathrm{sec}$ to $35 \mathrm{sec}$, a jet of water was directed to the specimen and the extent of descaling was assessed visually. A jet of water was obtained from a commercial spray nozzle, which was set just above the specimen at intervals of $110 \mathrm{~mm}$ so that all of the surface of the specimen was hit uniformly by the jet. Although industrial descaling is usually carried out by water jets with high pressures in the range about $100 \sim 250 \mathrm{~kg} / \mathrm{cm}^{2}$, water pressures of 1 , 4 and $15 \mathrm{~kg} / \mathrm{cm}^{2}$ were set up in this study because of the limitation arising from the apparatus used. Their flow rates were about 600,150 and $70 \mathrm{ml} / \mathrm{sec}$, respectively.

\section{Results}

\section{Growth Rate and Microstructure of Scale Film}

Thickness of scale films was calculated from the

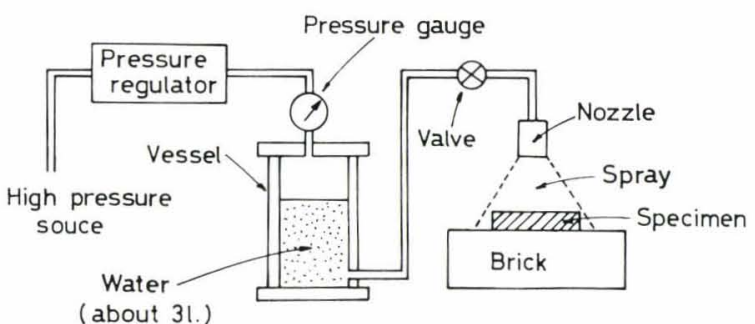

Fig. 1. Diagram of experiment on the hydraulic removal of scale films. oxidation weight gains. In the calculation, scale films were assumed to consist of wüstit ( $\mathrm{FeO}$ ), whose density is $5.7\left(\mathrm{~g} / \mathrm{cm}^{3}\right) .{ }^{27)}$ Thickness of scale films calculated from the weight gains was consistent approximately with those obtained from the microscopic study. They are shown in Fig. 2, which shows that the growth rate of scale films obeys the well-known parabolic rate law.

Microstructures of scale films cooled in air from the oxidation temperatures consisted of the wellknown three oxide layers, which were $\mathrm{Fe} / \mathrm{FeO} / \mathrm{Fe}_{3} \mathrm{O}_{4} /$ $\alpha-\mathrm{Fe}_{2} \mathrm{O}_{3}$. For examples, microstructures of scale films formed at $1000^{\circ}$ and $1100^{\circ} \mathrm{C}$ for $60 \mathrm{sec}$ are shown respectively in Photo. 1. These photographs show that $\alpha-\mathrm{Fe}_{2} \mathrm{O}_{3}$ layer (the outermost layer) is very thin and the greater part of scale films consists of $\mathrm{FeO}$ layer (the inner-most layer) and $\mathrm{Fe}_{3} \mathrm{O}_{4}$ layer (the middle layer). As the oxidation temperature increased, the relative thickness of $\mathrm{FeO}$ layer increased.

\section{Blistering of Scale Films}

In the preliminary study, preheating in vacuum was intended to decrease the formation of gas bubbles, such as $\mathrm{H}_{2}$ bubbles, released from the steel or iron, and pure iron with a small quantity of carbon

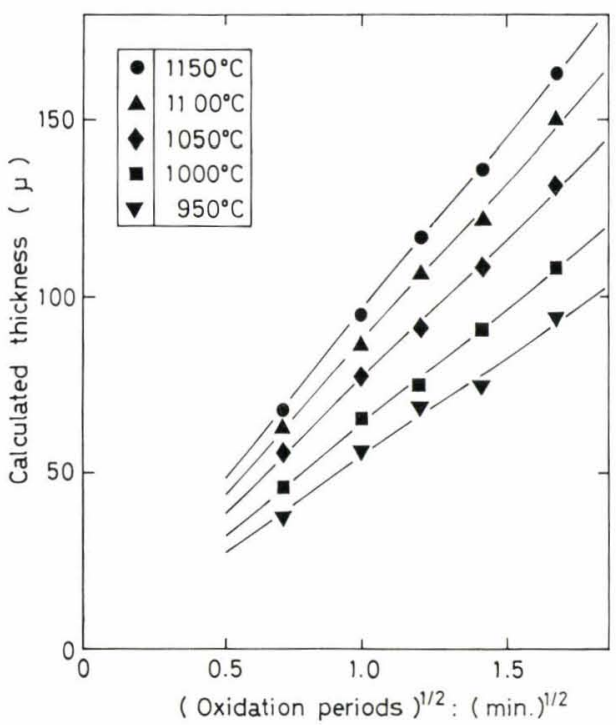

Fig. 2. Relation between the thickness of scale films and the oxidation periods. (a) oxidized at $1000^{\circ} \mathrm{C}$ for $60 \mathrm{sec}$

(b) oxidized at $1100^{\circ} \mathrm{C}$ for $120 \mathrm{sec}$

Photo. 1. Microstructures of scale films of rimmed steel.
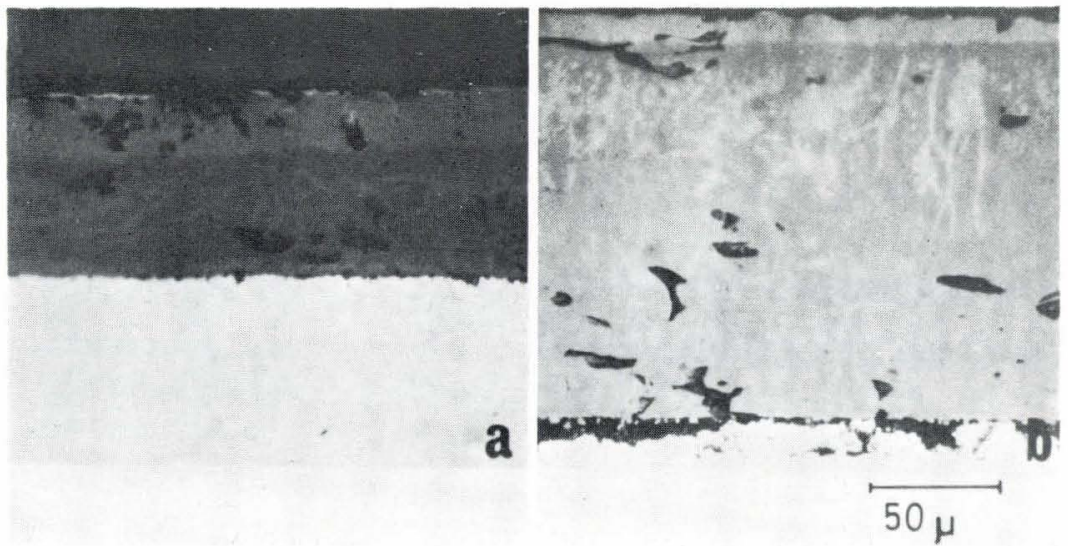
was used to decrease the formation of $\mathrm{CO}$ bubbles. Therefore, it was expected that, in case of specimens preheated in vacuum prior to oxidation, blisters did not appear, or decreased in number, if they were due to gas bubbles. Nevertheless, blistering was observed quite similarly in both specimens of the pure iron and the rimmed steel preheated in vacuum. Outward appearance of the pure iron during the oxidation is shown in Photo. 2. Blisters were small round spots as shown in Photos. 2 (b) and (c). They appeared about 25 30 sec after the start of oxidation and grew thereafter. The number and the incubation period from the start of oxidation to the appearance of blisters of preheated rimmed steel were approximately the same. The results of the preliminary study gave a suggestion that most of blisters observed in this study were caused by something other from gas bubbles.

Blistering was also observed to take place in the specimens which were covered individually by a steel sheet for the protection from the oxidation during the preheating in air. For a typical example, blistering of scale film of specimen oxidized during cooling from $1054^{\circ} \mathrm{C}$ is shown in Photo. 3. In this case, blisters of scale film appeared, as lots of spots, 8 sec after the start of oxidation and they grew individually. In a short time, they combined with some of adjacent ones and grew larger. Although blisters grew fast at the beginning, their growth rate decreased gradually.

Examination of the cross sections of blister showed that the blistered scale film detached itself from the steel at their boundary. A polished cross section of the blister is presented in Photo. 4 which shows that the length of the blistered scale film is apparently larger than that of the steel. The elongation of the scale film of one of the most developed blisters observed was about 7\%. Most of steel surfaces within blisters had interference colors such as blue or light brown owing to the oxide films which were too thin to be observed by the optical microscope. However, some of the steel surfaces within blisters, which were cooled carefully so as not to be damaged, had metallic luster. These observations showed that plastic deformation of the scale film occurred easily without suffering from any large damages at these high temperatures.

The incubation periods from the start of oxidation to the appearance of blisters were measured. They were almost the same, if the oxidation temperatures

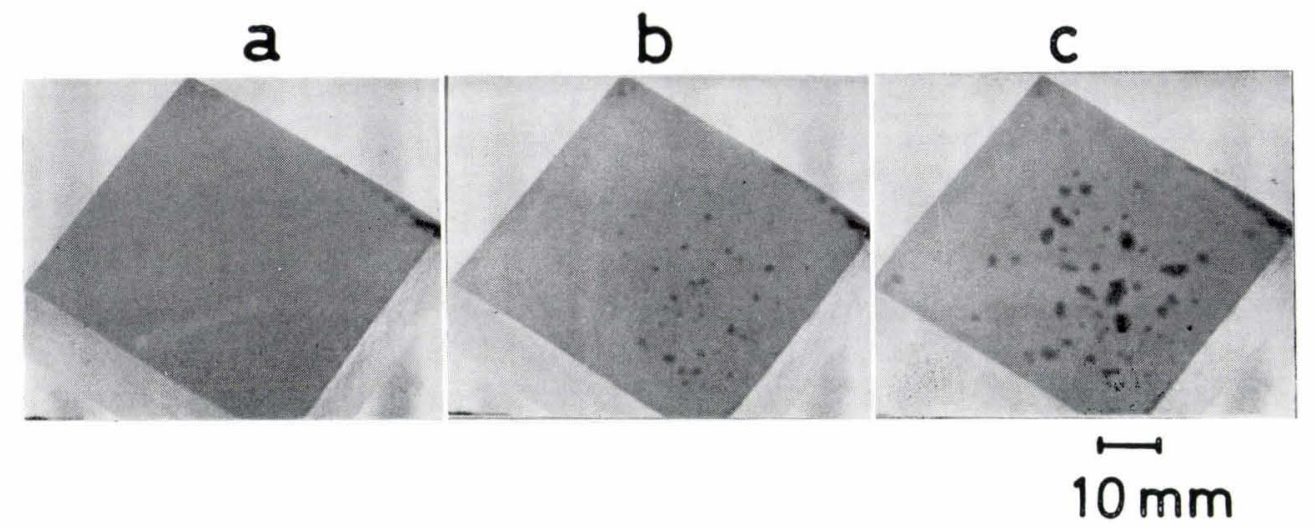

Photo. 2. Blistering of scale films of pure iron oxidized at $900^{\circ} \mathrm{C}$ for $15 \sec$ (a), $25 \sec$ (b) and $40 \sec$ (c) in air after the heat treatment in vacuum at $900^{\circ} \mathrm{C}$ for $1 \mathrm{hr}$.

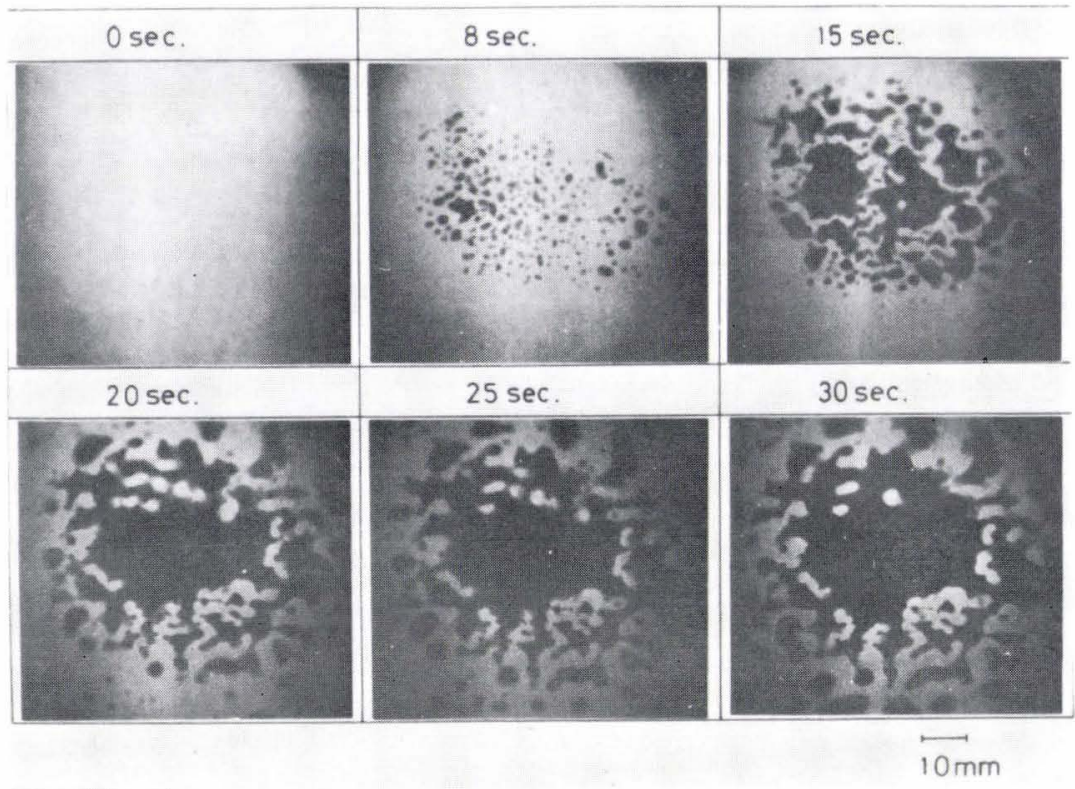

Photo. 3. Blistering of scale films during the air cooling from $1054^{\circ} \mathrm{C}$. 


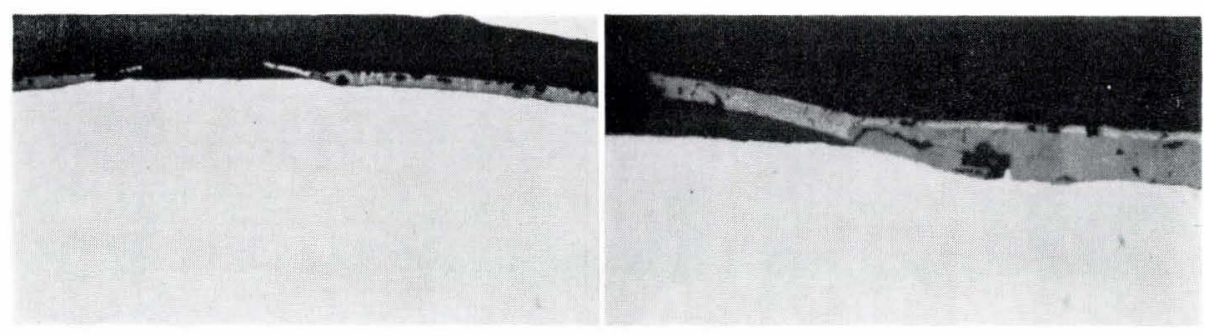

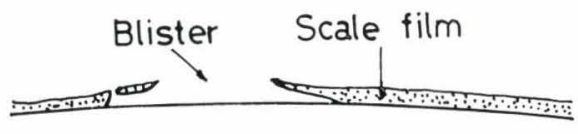

Steel

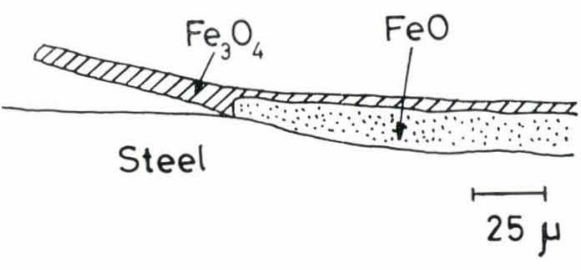

Photo. 4. Cross section of blistered scale formed during cooling from $950^{\circ} \mathrm{C}$.

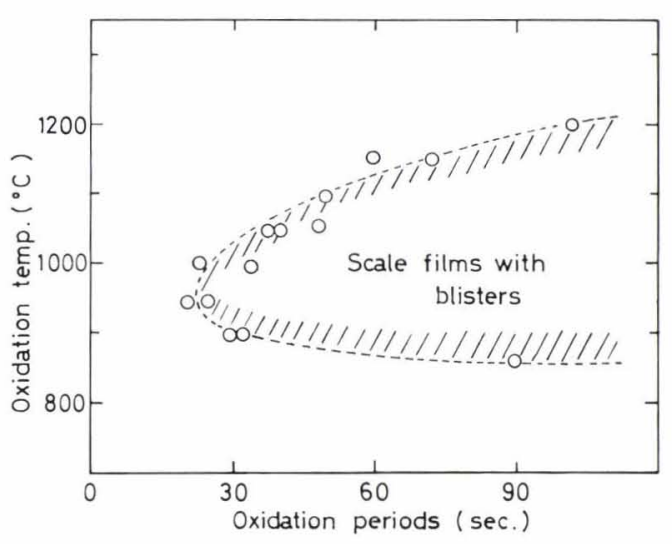

Fig. 3. Blistering behaviors of scale films in the isothermal oxidation.

were the same. The incubation periods at the isothermal oxidation were plotted vs. the respective oxidation temperatures and Fig. 3 was obtained. Figure 3 shows the followings: (1) At temperatures in the range $950^{\circ} \sim 1000^{\circ} \mathrm{C}$, blisters appeared after the shortest oxidation periods. (2) If the oxidation temperatures were apart from the above temperature range, the incubation periods became longer. (3) Blistering was not observed at temperatures below about $850^{\circ} \mathrm{C}$ or above about $1200^{\circ} \mathrm{C}$.

At temperatures in the range $950^{\circ}$ to $1000^{\circ} \mathrm{C}$, the growth rate of blisters was the largest.

The incubation periods at the oxidation during cooling were also measured and the results shown in Fig. 4 were obtained. In Fig. 4, the heat patterns and the incubation periods of individual specimens were also shown. The comparison between Fig. 3 and Fig. 4 indicates that the oxidation during cooling brings about the following differences:

(1) Temperature range, in which blisters appear, enlarges.

(2) The incubation periods become shorter on the whole.

(3) The nose temperature increases by about $100^{\circ} \mathrm{C}$.

It can be considered that these differences were due to the effects of thermal stress in addition to the

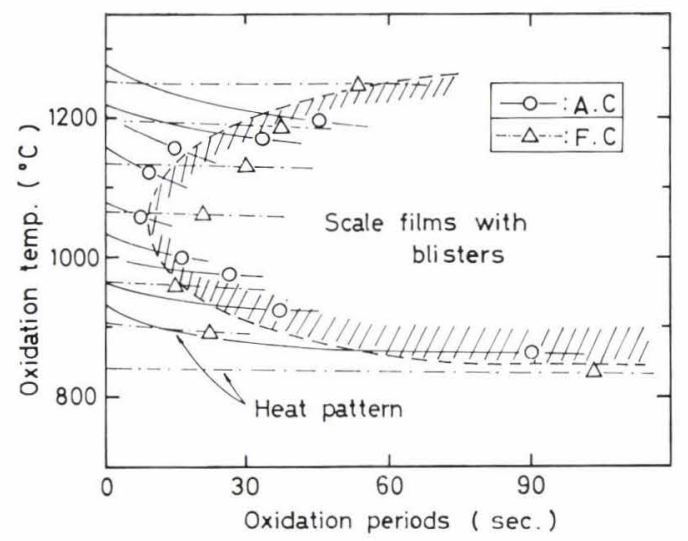

Fig. 4. Blistering behaviors of scale films in the oxidation during cooling.

oxidation stress.

In order to observe the effect of thermal stress more clearly thermal shock was given locally to some of the scale films, which were in contact with the steel, by spraying cold Ar gas. Photograph 5 shows the appearance of the specimen oxidized at $1200^{\circ} \mathrm{C}$ for $10 \mathrm{sec}$ and then cooled locally by Ar gas spraying. According to Fig. 4, it takes about $30 \mathrm{sec}$ for blisters to appear at $1200^{\circ} \mathrm{C}$. However, blisters appeared only $0.25 \mathrm{sec}$ after the start of Ar gas spraying. Moreover, they appeared at the position where jet of Ar gas was directed. As shown in Photo. 5, they increased rapidly in number and size in a short time. About $2.0 \mathrm{sec}$ later, they were observed on the almost all the surface. Although Ar gas spraying was carried out at various temperatures between $850^{\circ} \mathrm{C}$ and $1250^{\circ} \mathrm{C}$, blistering took place in the same manner as that shown in Photo. 5. The results mentioned above mean that thermal stress has a strong influences on the appearance of blisters.

In this study, the oxidation during the heating to the desired temperatures was prevented by covering one of surfaces of the specimen with a cold-rolled rimmed steel sheet. In order to examine the effect of the sheet cover, some of sheets, which were cooled rapidly in air after the removal from the specimen, were examined. The thickness of most of scale 


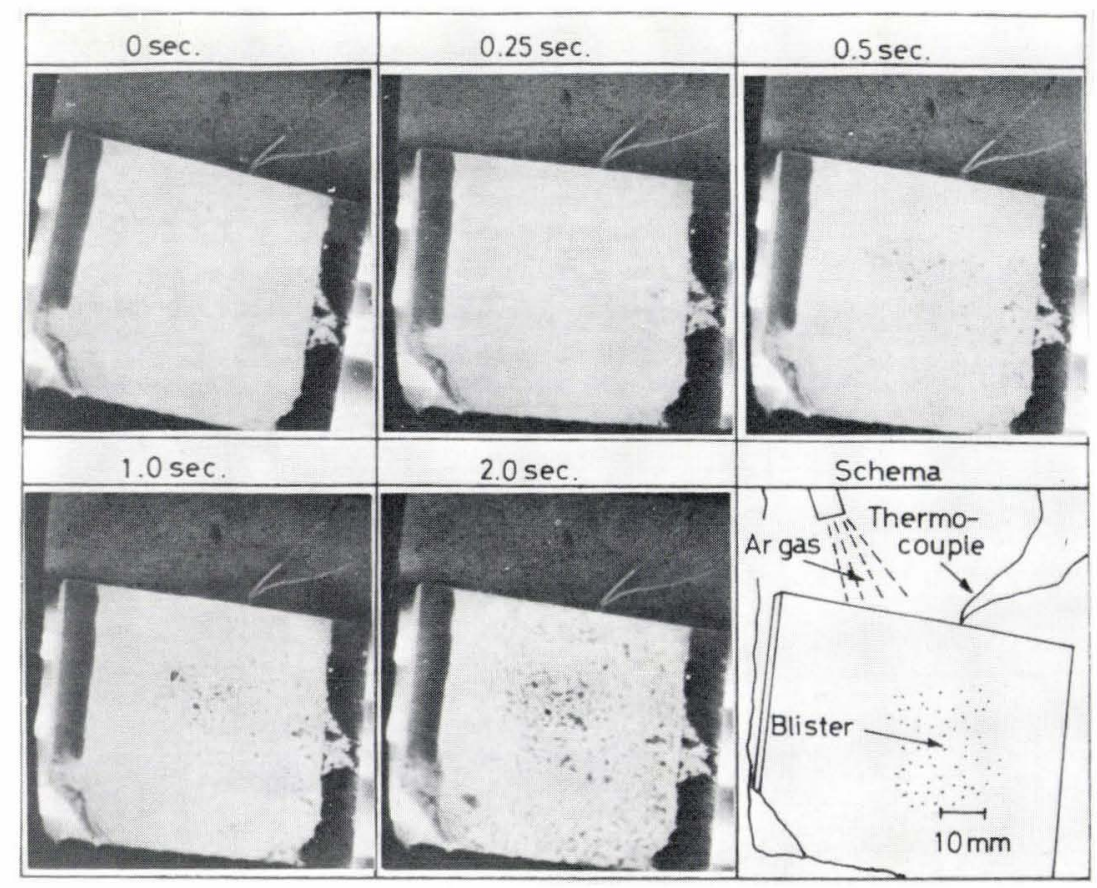

Photo. 5. Blistering of scale films by Ar gas spraying after the oxidation at $1200^{\circ} \mathrm{C}$ for $10 \mathrm{sec}$

films formed on the surfaces, which were in contact with the specimens, was about $1 \mu \mathrm{m}$. As the sheet covers examined were cooled in air, it was expected that scale films formed on the specimens during the heating to the desired temperatures were far less than $1 \mu \mathrm{m}$ thick.

\section{Descaling by Water Jet}

Scale films with some blisters were removed very easily by all the water jets used. In this case, blisters were broken readily by water jets and scale chips produced were subsequently washed away by the volume flow of water. Scale films being contact with the steel were undercut at the steel/scale interface and they bulged. Then, they were broken and washed away by water jet. These processes successively occurred mostly at the edge of the scale film fragments remained on the steel. It took about $0.3 \sim$ $0.5 \mathrm{sec}$ for all of scales on the specimen of size $100 \times$ $100 \mathrm{~mm}$ to be removed by the water jets with the pressure of 4 and $15 \mathrm{~kg} / \mathrm{cm}^{2}$. In case of water jet with the pressure of $1 \mathrm{~kg} / \mathrm{cm}^{2}$, about $1 \sim 3 \mathrm{sec}$ were required for the completion of descaling because the flow rate was so small (about $70 \mathrm{ml} / \mathrm{sec}$ ).

Removal behavior of scale films with no blisters varied depending on the water pressures used. Most of scale films with no blisters were generally difficult to be removed entirely by the water jets with the pressure of $15 \mathrm{~kg} / \mathrm{cm}^{2}$ although the local removal took place. However, scale films, just before the appearance of blisters, were removed by the water jet of the pressure of $4 \mathrm{~kg} / \mathrm{cm}^{2}$. When the water jet struk the scale films examined, blisters appeared locally and descaling proceeded in the same manner as that observed in the scale films with blisters. Blistering and subsequent descaling of scale films with no blisters, except for those formed at temperatures lower than about $900^{\circ} \mathrm{C}$, proceeded more easily in case of water jet with the pressure of $1 \mathrm{~kg} / \mathrm{cm}^{2}$. The size

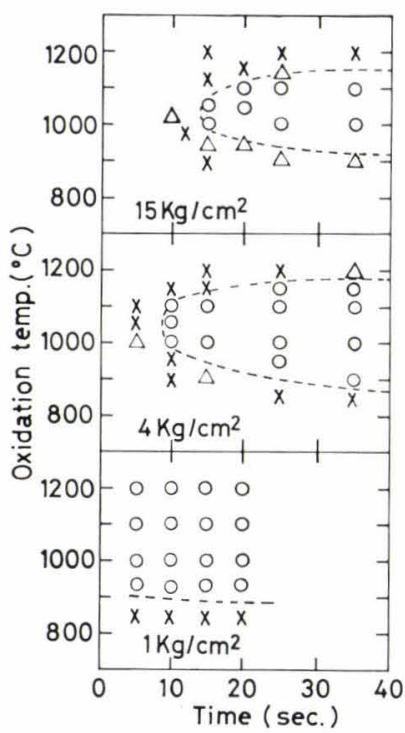

O: good, $\Delta$ : slightly bad, $\quad x$ : bad

Fig. 5. Results of hydraulic removal of scale films by the water jet with water pressures of 15,4 and $1 \mathrm{~kg} / \mathrm{cm}^{2}$.

of water drops of this jet was larger than that of the water jets with higher pressures. In this case, it was observed that a blister appeared at the point where one of drops struk. This phenomenon was quite similar to that observed during Ar gas spraying mentioned earlier.

The results of descaling mentioned above are summarized and shown in Fig. 5. It shows that the removal of scale films formed within a definite temperature-time range takes place easily. This range was, as mentioned above, dependent on the water pressure and it spread out as the pressure decreased. In case of the water jets with the pressures of 4 and $15 \mathrm{~kg} / \mathrm{cm}^{2}$, the definite ranges are comparatively similar to that where blistering takes place. This indicates that there is a strong relation between the 
blistering and the removability of scale films.

\section{Discussion}

\section{The Motive Force Causing Blistering}

The informations about blistering of scale films obtained in this study are summarized as follows:

(1) Blistering takes place even though the generation of gas bubbles is prevented as far as possible.

(2) Blistering occurs in the definite temperaturetime range.

(3) Changes of temperature during the oxidation have great influences on blistering. In case of the oxidation during cooling, the range where blistering takes place spreads out more than that in case of the isothermal oxidation.

Gas bubbles and the oxidation stress have been considered as the motive forces of blistering of scale films. ${ }^{25,26)}$ However, any positive evidences supporting that blisters are due to gas bubbles could not be obtained in this study. The summary (1) described above rather indicates the negative possibility. Moreover, it can be considered that the mechanism due to gas bubbles can not give any explanations of the temperature dependence of blistering shown in Figs. 3 and 4. Accordingly, it may be quite all right to consider that some motive forces other than gas bubbles should be considered for the explanation of blistering.

It was observed that blistering of scale films was promoted by Ar gas spraying or water drops impinging. These observations indicate clearly that the thermal stress has possibility of causing or promoting blistering. Although the detailed discussion is given later, it can be expected that the thermal stress may bring such situation where the oxide is under compression and the steel under tension. Accordingly, the same kind of stress should be expected as the motive force causing blisters in the scale films. On the basis of above discussion, it is concluded that most of blisters observed in this study were caused by the well-known oxidation stress, which has been elucidated ${ }^{19)}$ to bring the same stress situation as the thermal stress.

\section{On the Mechanism of Blistering of Scale Films}

For the generation of blisters, it is essential that the scale film is detached locally by the action of compressive stress and that the plastic deformation of the detached scale film takes place. It seems to be quite all right to consider that the latter condition is quite satisfied, because the scale film formed in this study consist mainly of wüstit which is known to have plasticity at high temperature. ${ }^{2,19,28)}$ Therefore, if the nuclei of blisters are formed, it can be expected that they grow through the plastic deformation of the detached scale films by the stress which developes at the neighborhood.

It has been observed earlier that the oxide phase can slip plastically on the metal surface during oxidation. ${ }^{19)}$ Modin' ${ }^{28)}$ has considered that this slip is caused by the oxidation stress. He confirmed this slip by observing the edges of the specimens where the two meeting oxide scales had prevented their mutual slip, thus forming clefts between steel and oxide scale. He has also considered that it is conceivable that, when wüstit phase slips over the steel surface, the contact between these phases is lost at certain points, thus forming pores.

These studies mentioned above indicate the possiblity of the nucleation of blisters by the compressive stress. If the area, where the contact is lost during the slip, is sufficiently large, it can be expected that such area acts as the nucleus of blister. This mechanism is considered to be quite probable although it is hard to say that the question is entirely solved.

One of the most important factors having influence on blistering is considered to be the adhesion of scale film to steel. On the adhesion of scale film to metal, several studies have been carried out. Tylecote ${ }^{291}$ studied the adherence of oxides on several metals after cooling from temperatures in the range $500^{\circ} \sim$ $1000^{\circ} \mathrm{C}$. He concluded that, as far as iron is concerned, purity is the most important factor in obtaining adherent films. Bruce and Hancock ${ }^{30}$ ) determined the thermal stress necessary to cause cracking in the scale and the subsequent breakdown of adhesion by cooling scaled surfaces rapidly. Their results indicated that the adhesive strength of scales on iron increased with increasing temperature from $570^{\circ} \mathrm{C}$ to $800^{\circ} \mathrm{C}$. Hulley and Rolles ${ }^{31)}$ studied high temperature cohesion and adhesion of oxide scales on mild steel containing $0.1 \% \mathrm{C}$. They showed that the adhesive strength of a scale/metal interface was dependent on temperature and scale thickness and that, at $1000^{\circ} \mathrm{C}$, the scale/metal minimum adhesive strength was about one third the value of the cohesive strength of the scale, which comprised of wüstit and magnetite. They also showed that the adhesive strength of scale formed at $850^{\circ} \mathrm{C}$ after $3 \mathrm{hr}$ oxidation in air was larger than that formed at $1000^{\circ} \mathrm{C}$ after $3 \mathrm{hr}$ oxidation. This was a trend which contrasted with that found by Bruce and Hancock. ${ }^{30}$ Tuck and Barlow ${ }^{32)}$ studied the adhesion of scale films to steels containing small quantities of Ni. They reported that Ni content and $\mathrm{O}$ content in the atmosphere used had a large effect on the adhesion. Their results indicated that the adhesion of a scale film formed at $1200^{\circ} \mathrm{C}$ was larger than that formed at $1100^{\circ} \mathrm{C}$. As described above, several studies have been reported on the problem of the adhesion. However, the problem seems to be still in a state of chaos. The informations obtained seem to be insufficient to estimate the adhesion of scale film to steel.

Figure 3 means that the time required for the breakdown of adhesion between scale and steel is dependent on temperature. This situation can be explained from the various combination of two factors, namely the stress and the adherence. However it is more satisfactory to consider that the contribution of the former towards making the situation is far greater than that of the latter because, judging from the results reported, ${ }^{30,31)}$ the latter can not be expected to have such severe temperature dependence enough 
to cause the situation as shown in Fig. 3. Accordingly, it can be considered that the oxidation stress developed in the scale film has the same trend as the incubation period. The following may be considered as the reason why the oxidation stress has the temperature dependence: The stress arises as a results of volume dilatation upon the conversion of metal to its oxide. Therefore, the development rate of the stress is expected to increase in proportion to the oxidation rate which increases with increasing temperature as shown in Fig. 2. However, it is also expected that the maximum value of the stress may be restricted by the stress relief through the plastic deformation of both wüstit ${ }^{19,28)}$ and steel, which takes place more easily with increasing temperature. It is satisfactory to consider that this must be the cause of the temperature dependence of the oxidation stress.

Above discussions can be summarized as shown in Fig. 6. The temperature dependence of the incubation period observed (right side) may be explained by the situation of the oxidation stress and the adherence (left side), assuming that blisters may appear at the time when the stress develops enough to break the adhesion between scale film and steel. The adhesive strength is tentatively considered to increase with increasing temperature as shown in Fig. 6. In case of the isothermal oxidation (Fig. 3), the incubation period is the shortest in the range $950^{\circ} \sim 1000^{\circ} \mathrm{C}$. This range may correspond to that where the oxidation stress reaches the highest value enough to cause the breakdown of the adhesion.

In case of the oxidation during cooling, thermally induced stress arises in addition to the oxidation stress. According to Goton, et al., ${ }^{33)}$ the linea expansion coefficient of wüstit is $14.0 \sim 14.1 \times 10^{-6}{ }^{\circ} \mathrm{C}^{-1}$ in the range $600^{\circ} \sim 1000^{\circ} \mathrm{C}$ and that of $\gamma-\mathrm{Fe}$ is $19.1 \times$ $10^{-6} \mathrm{C}^{-1}$ in the range $920^{\circ} \sim 1070^{\circ} \mathrm{C}$. This means that $\gamma$-Fe shrinks more than wüstit. Therefore, as far as the wüstite/steel interface is concerned, wüstit is under compression and steel under tension, when both phases are cooled with maintaining the contact. This situation is just the same as that of the oxidation stress. Accordingly, it is expected that thermal stress and the oxidation stress intensify mutually. As shown in Fig. 4, the temperature range and the

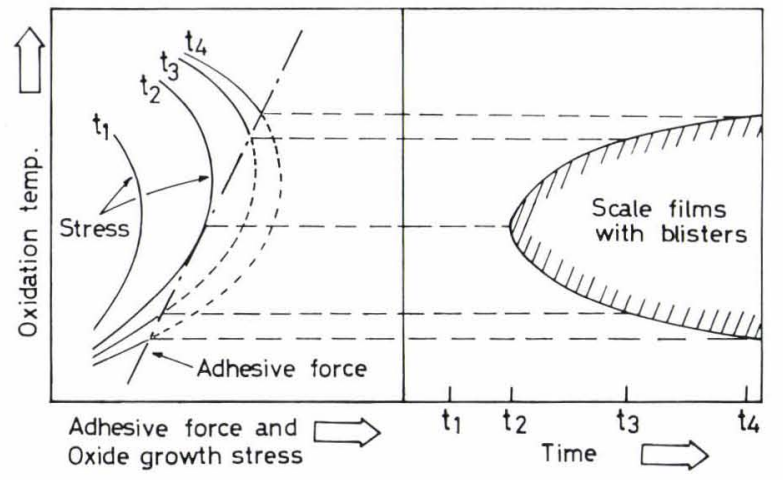

Fig. 6. Schematic illustrations of blistering behaviors in the isothermal oxidation. incubation periods changed into the direction that the action of the stress was strengthened. Moreover, blisters were observed to appear immediately after the local cooling either by Ar gas spraying or by water jet impinging. These results consist well with the expectation.

\section{Hydraulic Removal of Scale Film}

In this study, scale films with blisters, even though they were small in number, were removed readily by water jet. This indicates that the situation of the stress in the scale film is closely related with the hydraulic scale removal. It is satisfactory to consider that the adherent scale films formed within or near the formation range of blisters are just in the situation where the stress is barely smaller than the adhesion breakdown stress, as shown in Fig. 6 and that, according to this reason, they were removed readily by water jet because the stress is strengthened by the thermal stress. In case of scale films formed far away from the formation range of blisters, it can be considered that the development of the oxidation stress is insufficient to cause the breakdown of the adhesion, even though the thermal stress helps the action of the oxidation stress. However, good removal of scale films with no blisters was caused by the water jet with the pressure of $1 \mathrm{~kg} / \mathrm{cm}^{2}$. It should be emphasized that it took longer time with this water jet than with those of higher water pressures for the completion of descaling. It seems to be quite all right to consider that the differences between them may be caused by the following. In case of the former water jet, its cooling rate is comparatively small and suitable for causing the breakdown of the adhesion between scale film and steel approximately in the same way observed in Ar gas spraying. As compared with the former, the cooling rate of the latter water jets is so large that cracking of the scale film may be caused locally by the subsequent thermal stress and the continuous breakdown of the adhesion do not occur because of the stress relief through such cracking.

Sheppard and Steen $^{3 \prime}$ studied hydraulic descaling of steel by a water jet with various pressures from about 1 to $7 \mathrm{~kg} / \mathrm{cm}^{2}$. The hot-scaled slabs of size $150 \times 75 \times 12.5 \mathrm{~mm}$ heated in an electric-resistance furnace were examined. They showed that, though scale film below $200 \mu \mathrm{m}$ was removed, the thicker the scale film deposit at $1000^{\circ}$ and $1100^{\circ} \mathrm{C}$, the more difficult the oxide was to be removed. In this study, the thickness of scale films examined was less than about $100 \mu \mathrm{m}$ as shown in Fig. 2. Accordingly, the results obtained in this study are consistent with theirs.

Sheppard and Steen $^{3)}$ have also reported the sequence of scale removal as follows:

(i) The scale is undercut at the steel/scale interface.

(ii) Mosaic cracking occurs on planes normal to the interface, producing scale chips which are subsequently re moved by the volume flow of water. These observations are also consisted with those obtained in this study. 
In most of hot strip mills, descaling is usually carried out by a water jet with the pressure in the range $100 \sim 250 \mathrm{~kg} / \mathrm{cm}^{2}$. Accordingly, it would seem likely that commercial descaling depends mainly on mechanical removal after surface crazing by thermal stress. However, the results obtained in this study indicate that, if the condition is suitable, scale film can be removed sufficiently by a water jet with far lower pressure. It should be emphasized that the formation range of blisters should be used even for commercial hydraulic descaling.

\section{Conclusion}

In order to study the properties of scale films formed during hot rolling, rimmed steel plates were heated under the protection from the oxidation and oxidized in air for very short periods. Oxidation rate was studied at temperatures in the range $950^{\circ} \sim 1150^{\circ} \mathrm{C}$ for less than $180 \mathrm{sec}$. Blistering of scale film was observed at temperatures in the range $800^{\circ} \sim 1250^{\circ} \mathrm{C}$ and the situation of the stress in the scale film was presumed from the blistering behavior. Hydraulic removability of scale films formed at temperatures in the range $800^{\circ} \sim 1200^{\circ} \mathrm{C}$ was examined by water jets with the pressures of 1,4 and $15 \mathrm{~kg} / \mathrm{cm}^{2}$.

Results obtained are summarized as follows.

(1) Growth rate and microstructure of scale films

Scale films formed within $180 \mathrm{sec}$ had the wellknown three layered structures of $\mathrm{Fe} / \mathrm{FeO} / \mathrm{Fe}_{3} \mathrm{O}_{4} / \alpha$ $\mathrm{Fe}_{2} \mathrm{O}_{3}$ and they grew according to the parabolic rate law.

(2) Blistering of scale films

In the case of the isothermal oxidation, blistering was observed at temperatures in the range about $850^{\circ} \sim 1200^{\circ} \mathrm{C}$. In the range $950^{\circ} \sim 1000^{\circ} \mathrm{C}$, blistering took place after the shortest incubation periods. It was satisfactory to consider that, in this case, blisters were caused by the oxidation stress.

In the case of the oxidation during cooling, blistering took place more easily because of the influences of the thermal stress.

(3) Hydraulic removal of scale films

Scale films formed in the range where blistering occurred were removed readily by a water jet. This indicated that the stress in the scale film was closely related to the hydraulic removal.

\section{Acknowledgements}

The author wishes to express his gratitude to Dr. N. Oda, Managing Director, Manager of Central Research Laboratories of Sumitomo Metal Industries, Ltd. for the permission to publish the present paper.
He also wishes to acknowledge Dr. T. Shiraiwa, Assistant Manager of Central Research Laboratories of Sumitomo Metal Industries, Ltd. for the encouragement and guidance extended to him.

\section{REFERENCES}

1) O. Kubaschewski and B. E. Hopkins: Oxidation of Metals and Alloys, Academic Press, New York, (1962).

2) K. Hauffe: Oxidation of Metals, Plenum Press, New York, (1965).

3) T. Sheppard and W. M. Steen: JISI, 208 (1970), 797.

4) N. B. Pilling and R. E. Bedworth: J. Inst. Metals, 29 (1923), 529

5) U. R. Evance: Inst. Metals Symp. on Internal Stresses in Metals and Alloys, London, (1947), 219.

6) D. D. Dankov and P. V. Churaev: Dokl. Akad. Nauk SSSR, 73 (1950), 1221.

7) D. L. Douglass: Oxid. Metals, 1 (1969), 127.

8) J. Stringer: Corros. Sci., 10 (1970), 513.

9) R. E. Pawel and J. J. Cambell: Acta Met., 14 (1966), 1827.

10) W. K. Appleby and R. F. Tylecote: Corros. Sci., 10 (1970), 325 .

11) C. Roy and B. Burgess: Oxid. Metals, 2 (1970), 235

12) F. N. Rhines and J. S. Wolf: Met. Trans., 1 (1970), 1701.

13) W. Jaenicke, S. Leistikow and A. Stadler: J. Electrochem. Soc., 111 (1964), 1031.

14) W.J. Moore: J. Chem. Phys., 21 (1953), 1117.

15) R. Darras, D. Leclereq and R. David: J. Nucl. Mat., 8 (1963), 23

16) J. Stringer: J. Less-Common Metals, 16 (1968), 55.

17) J. D. Noden, C.J. Knights and M. W. Thomas: Brit. Corros. J., 3 (1968), 47.

18) J. E. Antill, M.J. Bennett, G. H. Chaffey, K. A. Peakall and J. B. Warburton: J. Nucl. Mat., 36 (1970), 1.

19) H. Engell and F. Wever: Acta Met., 5 (1957), 695.

20) J. E. Harris: Acta Met., 26 (1978), 1033.

21) M. V. Speight and J. E. Harris: Acta Met., 26 (1978), 1043.

22) C. Wagner: Z. Physik. Chem., 21B (1933) 25.

23) F. A. Golightly, F. H Stoot and G. C. Wood: Oxid. Metals, 10 (1976), 163.

24) Kinzokubinran, ed. by The Japan Institute of Metals, Maruzen, Tokyo, (1971), 1586.

25) M. Sugiyama: Kinzoku Zairyo no Kanetsu to Sanka, Seibundo Shinkosha, Tokyo, (1955), 68.

26) S. Modin, and E. Tholander: Jernkont. Ann., 144 (1960), 813.

27) G. V. Samsonov: The Oxide Handbook, IFI/PLENUM. New York, (1973), 34.

28) S. Modin: Met. Treatment, 29 (1962), 89.

29) R. F. Tylecote: JISI, 196 (1960), 135.

30) D. Bruce and P. Hancock: JISI, 208 (1970), 1021.

31) J. M. Hulley and R. Rolles: JISI, 208 (1970), 1029.

32) C. W. Tuck and J. Barlow: Iron Steel, (1972), No. 2, 31.

33) A. T. Gorton, G. Bitsianes and T. L. Joseph: Trans. Met. Soc. AIME, 233 (1965), 233. 